\title{
EXPERIMENT OF RF VOLTAGE MODULATION AT SRRC
}

\author{
M.H. Wang*, Peace Chang, P.J. Chou, K.T. Hsu, C.C. Kuo, J.C. Lee, W.K. Lau, \\ SRRC, Hsinchu, Taiwan
}

\section{Abstract}

The effects of amplitude modulation of RF cavity voltage on the longitudinal beam dynamics were studied experimentally at SRRC by using a streak camera system. The characteristics of parametric resonance in single bunch beam was investigated both by simulation and measurement. The formation of beamlets in the bunch helps to damp the coherent bunch oscillation. This property is employed to provide stable beam in user's shift at SRRC.

\section{INTRODUCTION}

The modulation of RF system is one of the important beam dynamics topics in accelerator physics. More and more accelerator physicists use the nonlinear property of parametric resonance of RF field modulation intentionly as an advantage to improve the performance of the accelerator[1]. The parametric resonance of RF field modulation resulted in redistribution of particles in the longitudinal plane. The formation of islands within an RF bucket acceptance reducing the density in the bunch core. The beam dynamics property related to the bunch density such as beam life time, beam instability etc. can be improved. At SRRC the effect of the RF phase modulation and the effect of RF voltage modulation were studied. The study of RF phase modulation at SRRC was reported elsewhere[2]. In this paper we discuss the longitudinal beam dynamics with RF voltage modulation. A simulation program was written to track the particle distribution. The characteristics of single bunch under parametric resonance were measured by using streak camera. The application and effects on multibunch is also reported.

\section{LONGITUDINAL BEAM DYNAMICS WITH RF VOLTAGE MODULATION}

For a charge particle in a circular accelerator, when the amplitude of the RF cavity voltage is modulated by a sinusoidal wave with amplitude $\epsilon$ in unit of RF cavity operational voltage and frequency $f_{m} \mathrm{~Hz}$ or modulation tune $\nu_{m}=\frac{2 \pi f_{m}}{\omega_{0}}$, where $\omega_{0}$ is the revolution angular frequency, the equation of motion are given by

$$
\begin{aligned}
\frac{d \phi}{d \theta}= & \nu_{s} \delta \\
\frac{d \delta}{d \theta}= & \frac{h \eta}{2 \pi E_{0} \nu_{s}}\left[e V_{R F}\left(1+\epsilon \sin \left(\nu_{m} \theta+\xi\right)\right)\right. \\
& \left.\sin \left(\phi_{s}+\phi\right)-U\right]
\end{aligned}
$$

Where $\theta=\omega_{0} t$ is the revolution angle, $\phi_{s}$ the synchronous phase, $\delta$ defined by $\frac{h \eta}{\nu_{s}} \frac{\epsilon}{E_{0}}$ with $\eta$ the slip factor, $h$ the harmonic number, and $U$ the radiation energy loss, which

\footnotetext{
*Email:mhwang@srrc.gov.tw
}

depends on the energy of the particles. The synchrotron mapping equation are given by

$$
\begin{aligned}
\phi_{n+1}= & \phi_{n}+2 \pi \nu_{s} \delta_{n} \\
\delta_{n+1}= & \delta_{n}-2 \pi \nu_{s}\left[1+\epsilon \sin \left(\nu_{m} \theta+\xi\right)\right] . \\
& \sin \left(\phi_{s}+\phi_{n+1}\right)-\frac{4 \pi \alpha}{\omega_{0}} \delta_{n}
\end{aligned}
$$

Where $\alpha$ is the radiation damping factor, which is about $200 s^{-1}$ of storage ring at SRRC for $1.5 \mathrm{GeV}$. Comparing to the synchrotron angular frequency $\omega_{s} 1.6 \times 10^{5} s^{-1}$, it is smaller. Neglecting damping term and the corresponding Hamiltonian is

$$
H=\frac{1}{2} \nu_{s} \delta^{2}+\nu_{s}\left[1+\epsilon \sin \left(\nu_{m} \theta+\xi\right)\right][1-\cos \phi]
$$

Analyzing the Hamiltonian we derive island structure in the phase space. The results of applying modulation frequency two times around the synchrotron frequency is summarized[3]. The stable fixed points(SFP's) are located at $J_{S F P}=8\left(1-\frac{f_{m}}{2 f_{s}}\right)+2 \epsilon$ for $=0$ and $=\pi$, the unstable fixed points(UFP's) located at $J_{U F P}=8(1-$ $\left.\frac{f_{m}}{2 f_{s}}\right)-2 \epsilon$ for $f_{m} \leq 2 f_{s}-\frac{1}{2} \epsilon f_{s}=f_{2}$, or $J_{U F P}=0$ for $f_{2} \leq f_{m} \leq 2 f_{s}+\frac{1}{2} \epsilon f_{s}=f_{1}$ for $=\frac{\pi}{2}$, $\frac{3 \pi}{2}$. A tracking program by using Eq.(3) and Eq.(4) is written. Several cases represent the $f_{m}<f_{2}, f_{m}=f_{2}, f_{2}<$ $f_{m}<f_{1}, f_{m}=f_{1}$ and $f_{m}>f_{1}$ are investigated. The parameters of storage ring at SRRC are used for tracking. The synchrotron frequency of the ring is $25.45 \mathrm{kHz}$, the damping rate is $200 s^{-1}$, and the revolution frequency is $2.5 \mathrm{MHz}$. The modulation amplitude $\epsilon$ is 0.1 and the corresponding $f_{2}$ is $49.6275 \mathrm{kHz}$ and $f_{1}$ is $52.1725 \mathrm{kHz}$. The initial particles are uniformly distributed $100 \times 100$ in a rectangular box of dimension $\phi \in[-\pi, \pi], \delta \in$ $[-2,2]$. The results of tracking of 25000 turns are shown in Fig. 1. As the modulation frequency increases from low frequency to high frequency, the two outer islands move towards the center core, and the population in center core decreases and the two outer islands increases. As modulation frequency equals $f_{2}$ the center core disappears. As the modulation increases further the two outer islands come closer, at modulation frequency equals $f_{1}$ the two outer islands merge and there exists again single beamlet in a RF bucket. The two outer islands rotate around the center of the RF bucket with angular frequency equals to one half the modulation frequency in all the process.

\section{EXPERIMENTAL RESULTS}

The experiments of RF amplitude modulation is operated at single bunch mode with beam current about $2 \mathrm{~mA}$. A sinusoidal wave from HP 33120A function generator was split into two to apply to the two gapvoltage feedback loop 

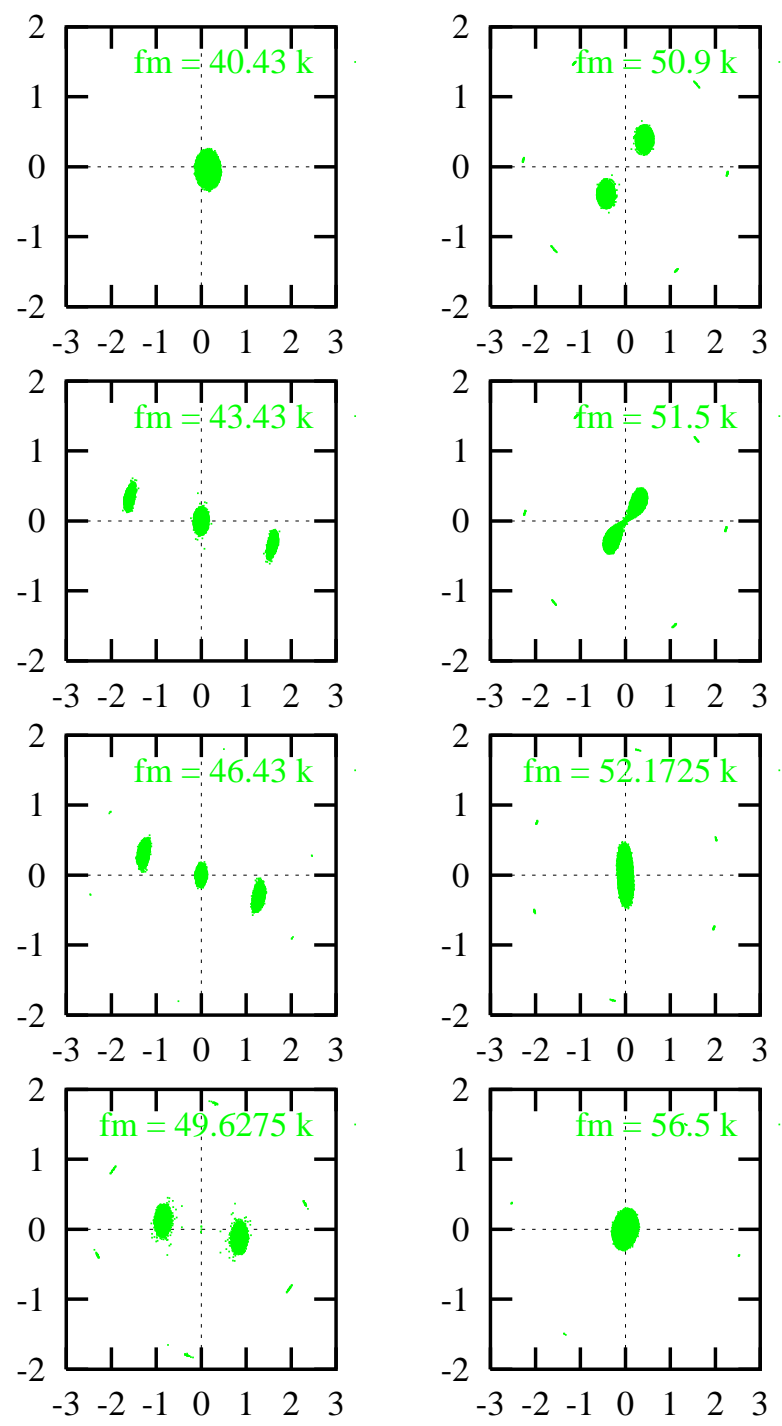

Figure 1: Simulation results of RF amplitude modulation. The modulation frequency is increased from top to down and left to right. The modulation amplitude is $10 \%$ of the cavity voltage.

of RF cavity low level system respectively. The operational gapvoltage of each cavity is $400 \mathrm{kV}$. The modulation frequency is increased step by step from low frequency below $2 f_{s}$ to high frequency above $2 f_{s}$. The modulation amplitude ratio is 0.083 and 0.066 respectively. The results were recored by the streak camera. The formation of three beamlets in a RF bucket is shown in Fig. 2. The modulation frequency was $51.08 \mathrm{~K} \mathrm{~Hz}$ with modulation amplitude ratio 0.083. From the upper part of the Fig. 2 it shows that two outer islands rotate with the bucket center at frequency one half the modulation frequency. The lower part of the Fig. 2 shows three beamlets in a single bunch. Which is predicted by the theoretical analysis at modulation frequency below $f_{2}$. The theoretical value of $f_{2}$ in this case is $49.84 \mathrm{~K}$ $\mathrm{Hz}$. However the observation revealed even at modulation frequency higher than $51.08 \mathrm{kHz}$ there could exit three beamlets in a single bunch. The reason for this discrepancy may be due to the non-coincidence when applied the modulation to the double RF system. The non zero single bunch beam current may affect the results too. It was also found the frequency response was different when modulation frequency was swept from low to high and from high to low. The phenomenon is like the hysteresis of magnetic field.
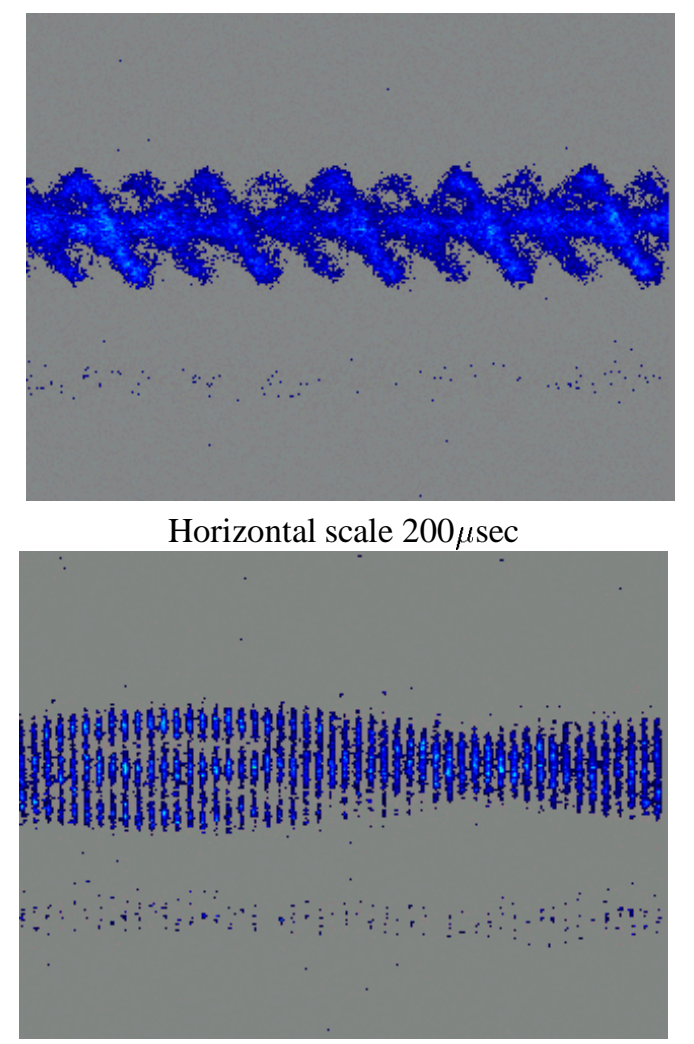

Horizontal scale $10 \mu \mathrm{sec}$

Figure 2: Formation of islands in single bunch beam under $\mathrm{RF}$ voltage modulation. The modulation frequency is 51.08 $\mathrm{kHz}$ and the amplitude is $8.3 \%$ of the RF cavity voltage. The upper plot shows the two outer islands rotate with the bucket center at frequency one half the modulation frequency. The lower part shows the structure in a bunch and the turn by turn motion. The full vertical scale is 1.4 ns.

\section{SUPPRESSION OF BEAM INSTABILITY}

Longitudinal coupled bunch beam instability is one of the beam instabilities, which deteriorate the beam property. A longitudinal bunch by bunch feed back system is implemented and under commissioning to suppress the instability [4]. Before the completion of the longitudinal feed back system the RF amplitude modulation is used as a temporary solution to suppress the longitudinal coupled bunch beam instability. A modulation frequency slightly below two times of synchrotron frequency with $10 \%$ amplitude modulation was applied to the RF system. A 
beam spectrum measured from the BPM sum signal from HP4396A before and after the modulation is shown in Fig. 3. The intensity of the beam spectrum is largely reduced after applying the modulation. The suppression of synchrotron side band near revolution harmonics is also shown in Fig. 4. In Fig. 4 the two revolution harmonics is complementary to multiplier of harmonics numbers. The multibunch beam motion under RF amplitude modulation was also recorded by streak camera. The result is shown in Fig. 5. The picture is much like the single bunch event except the amplitude of motion is larger. This indicates that the rotations of beamlets within all the bunches are in phase.

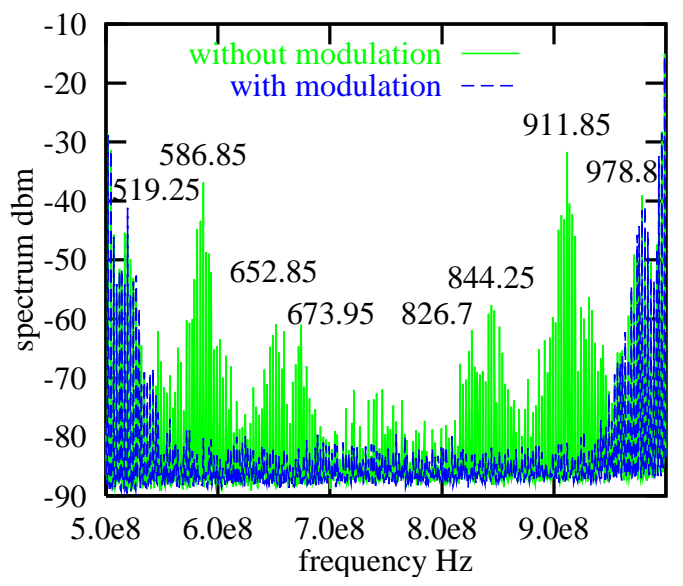

Figure 3: Beam spectrum from BPM sum signal before and after applying RF amplitude modulation. The modulation frequency was $50.155 \mathrm{kHz}$ and the amplitude modulation is $10 \%$. The frequency span of the spectrum is $500 \mathrm{MHz}$.

In order to further investigate the capability of suppression of beam instability by RF amplitude modulation, the sextupole strength of normal operation was reduced and the vertical betatron side had appeared. The synchrotron photon image pulsing on the screen monitor. When applying the modulation the vertical betatron side band was suppressed and the synchrotron photon image was stabilized again as shown on the screen monitor. From this study it shows the beam can be stabilized by the RF voltage modulation even at lower sextupole strength which is usually to be set to a higher value to provide the head-tail damping.

The application of the RF amplitude modulation close resonance will reduce line charge density, provide larger energy spread. Thus suppresses the coherent motion of beam and increase lifetime. However, because the increase of energy spread the horizontal beam size at dispersion region will increase.

\section{REFERENCES}

[1] J.D. Fox, et al.,EPAC'92, proceedings, p. 1079, P. Kuske, EPAC' 98, proceedings, Yu, Senichev, et al., EPAC' 98, proceedings.

[2] M.H. Wang, et al, EPAC'97, proceedings.
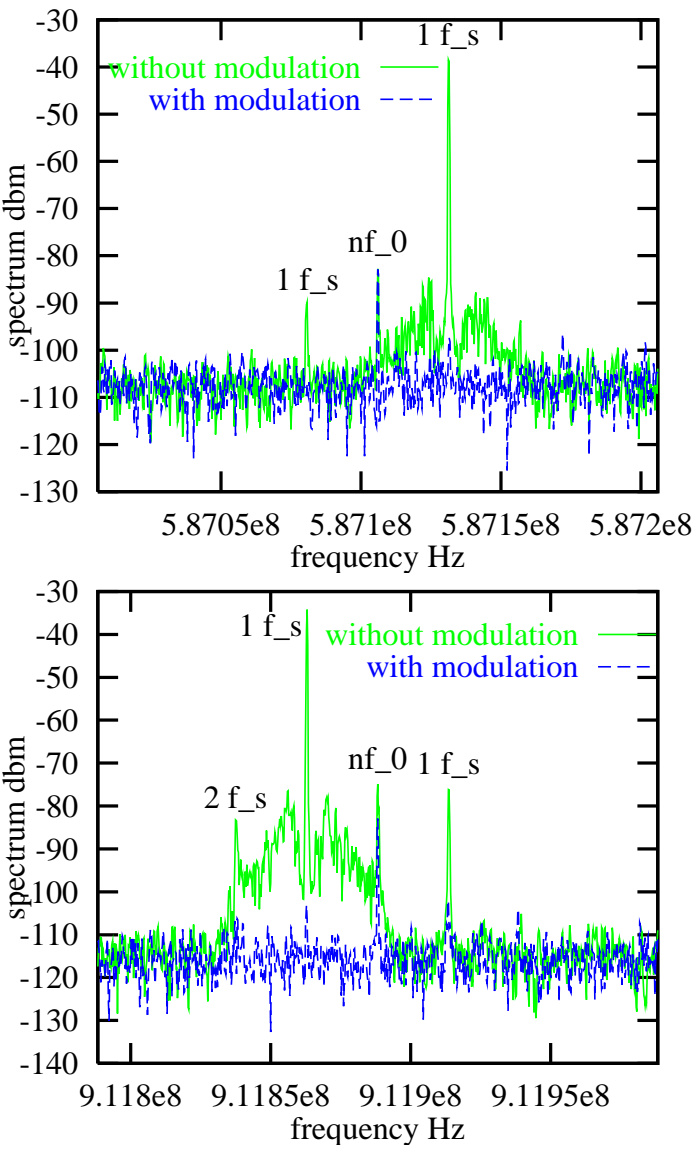

Figure 4: Beam spectrum zoom in from Fig. 3. The revolution harmonic frequency of the upper is 587.106 $\mathrm{MHz}$, and the lower is $911.888 \mathrm{MHz}$. The frequency span of the spectrum is $200 \mathrm{kHz}$.

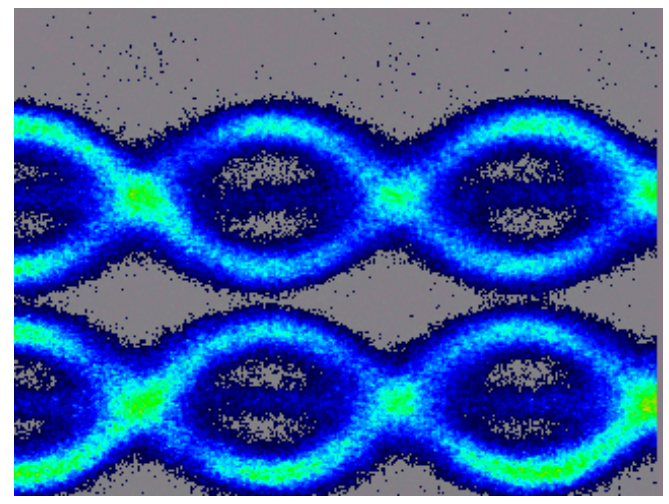

Horizontal scale $100 \mu \mathrm{sec}$

Figure 5: Longitudinal motion of multibunch beam under $\mathrm{RF}$ amplitude modulation. The modulation frequency is $50.8 \mathrm{kHz}$ and the amplitude is $8.3 \%$ of the RF voltage. The full vertical scale is $1.4 \mathrm{~ns}$ and $100 \mu \mathrm{sec}$ for horizontal.

[3] D. Li, et al., Phys. Rev. E 48, p. 1638(1993). D. Li, et al., Nucl. Instr. and Meth. A364(1995)205.

[4] W.K. Lau, et al., these proceedings. 